\title{
MISALIGNMENT PERFORMANCE OF SELECTIVE TAP ADAPTIVE ALGORITHMS FOR SYSTEM IDENTIFICATION OF TIME-VARYING UNKNOWN SYSTEMS
}

\author{
Patrick A. Naylor, Andy W.H. Khong and Mike Brookes \\ Department of Electrical and Electronic Engineering, Imperial College London, UK
}

\begin{abstract}
Selective tap algorithms have been proposed as a means of reducing complexity for adaptive filtering. MMax tap selection has been employed in many algorithms due to its straightforward implementation. This paper formulates the analysis of two MMax-based algorithms under time-varying unknown system conditions as are often found in practical applications. The steady-state misalignment for the MMax normalized least mean square and the MMax recursive least squares algorithms are derived and their performance is compared to that of their respective full-update algorithms. The tradeoff between computational complexity and misalignment performance is also shown for the MMax normalized least mean square case.
\end{abstract}

Index Terms - Acoustic echo cancellation, Partial update adaptive filtering, Misalignment performance

\section{INTRODUCTION}

Adaptive filters are widely used in many applications of signal processing. The normalized least-mean-square (NLMS) algorithm [1] is common in practice due to its straightforward implementation and relatively low complexity. The recursive least squares (RLS) is better performing but requires substantially more computation. The demands made of adaptive filters by the deployment of new technologies call for ever-increasing performance, modelling of longer impulse responses and lower computational complexity for system identification applications such as acoustic echo cancellation (AEC) as shown in Fig1.

A result of efforts to reduce the complexity of adaptive algorithms is a class of selective tap adaptive algorithms that share the characteristic of executing tap update operations on only a subset of the filter coefficients at each iteration. This can be achieved by selecting the taps using one of several criteria. Examples include decimation in the space of the tap-update vector such as in SequentialLMS [2], temporal decimation such as in Periodic-LMS [2], imposition of a sparse approximation to the tap-update vector such as in MMax normalized least-mean-square (MMax-NLMS) [3], MMax recursive least squares (MMax-RLS) [4] and Selective Partial-update algorithms [5] that can be built around both NLMS and the affine projection algorithm [6]. Although partial-update algorithms were originally proposed to address computational complexity issues as has been discussed, a class of selective-tap algorithms have also been applied to stereophonic AEC [4][7] giving improved performance.

Important performance measures for adaptive filters characterize the initial convergence rate, the residual error after convergence, the ability to track time-varying systems and the computational complexity. This paper focuses on the steady-state misalignment performance of MMax-NLMS and MMax-RLS algorithms when tracking non-stationary systems. Consideration of algorithms' performance

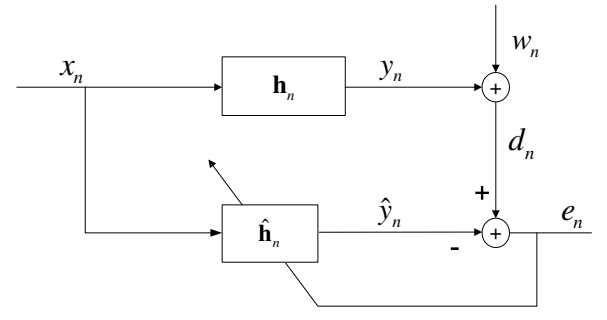

Fig. 1. System identification structure.

under such dynamic conditions is important since the unknown system is often non-stationary in practical applications. It is therefore necessary to include a time-varying system model in the analysis of such adaptive algorithms as indicated in several studies including [8][9]. Although performance in terms of mean square error has been analyzed for the MMax-NLMS algorithm [3], this considered only stationary systems. Our new analysis describes the performance of a generalized class of adaptive algorithms, including both fully updating and selective tap updating for non-stationary unknown systems. This work considers the trade-off between computational complexity and misalignment under such non-stationary unknown system conditions.

\section{THE MMAX-NLMS AND MMAX-RLS ALGORITHMS}

In this section, we review the MMax-NLMS and MMax-RLS algorithms. Figure 1 shows a system identification structure in which, at the $n^{\text {th }}$ iteration, $y_{n}=\mathbf{x}_{n}^{T} \mathbf{h}_{n}$ and the tap-input vector $\mathbf{x}_{n}=\left[x_{n}(0), \ldots, x_{n}(L-1)\right]^{T}$ while the unknown system, $\mathbf{h}_{n}=\left[h_{n}(0), \ldots, h_{n}(L-1)\right]^{T}$ and $L$ is the length of $\mathbf{h}_{n}$. An adaptive filter $\widehat{\mathbf{h}}_{n}=\left[\widehat{h}_{n}(0), \ldots, \widehat{h}_{n}(L-1)\right]^{T}$ is used to estimate $\mathbf{h}_{n}$ by adaptively minimizing the error signal $e_{n}=y_{n}+w_{n}-\hat{y}_{n}$, where the $\widehat{y}_{n}=\mathbf{x}_{n}^{T} \widehat{\mathbf{h}}_{n}$ and $w_{n}$ is measurement noise.

\subsection{The MMax-NLMS algorithm}

In the MMax-NLMS algorithm [10], only those taps corresponding to the $M$ largest magnitude tap-inputs are selected for updating at each iteration. Defining the subselected tap-input vector $\widetilde{\mathbf{x}}_{n}=\mathbf{Q}_{n} \mathbf{x}_{n}$ where $\mathbf{Q}_{n}=\operatorname{diag}\left\{\mathbf{q}_{n}\right\}$ is a $L \times L$ tap selection matrix and $\mathbf{q}_{n}=\left[q_{n}(0), \ldots, q_{n}(L-1)\right]^{T}$, elements $q_{n}(i)$ for $i=0,1, \ldots, L-1$ is given by,

$$
q_{n}(i)=\left\{\begin{array}{ll}
1 & \left|x_{n}(i)\right| \in\left\{M \text { maxima of }\left|\mathbf{x}_{n}\right|\right\} \\
0 & \text { otherwise }
\end{array},\right.
$$

where $\left|\mathbf{x}_{n}\right|=\left[\left|x_{n}(0)\right|, \ldots,\left|x_{n}(L-1)\right|\right]^{T}$. Defining $\|\cdot\|_{2}^{2}$ as the squared $l_{2}$-norm, the MMax-NLMS tap-update equation is then given by

$$
\widehat{\mathbf{h}}_{n+1}=\widehat{\mathbf{h}}_{n}+\mu \frac{\mathbf{Q}_{n} \mathbf{x}_{n} e_{n}}{\left\|\mathbf{x}_{n}\right\|_{2}^{2}+\delta},
$$


where $\delta$ and $\mu$ are the regularization parameter and step-size respectively. To select the $M$ maxima of $\left|\mathbf{x}_{n}\right|$, MMax-NLMS employs the SORTLINE algorithm [11]. The computational complexity in terms of multiplications and sorting operations for MMax-NLMS is $\mathcal{O}\left(L+M+2 \log _{2} L\right)$ compared to $\mathcal{O}(2 L)$ for NLMS. Further reduction in sorting operations can be achieved using the Short-sort algorithm [12].

\subsection{The MMax-RLS algorithm}

The MMax-RLS algorithm [4] sub-samples the tap-input vectors at each time iteration based on the MMax tap selection criterion given in (1) where the subselected tap-input vectors propagate consistently through the memory of the RLS algorithm. Defining $\widetilde{\mathbf{x}}_{n}=\mathbf{Q}_{n} \mathbf{x}_{n}$, the MMax-RLS tap-update equation is given by

$$
\widehat{\mathbf{h}}_{n+1}=\widehat{\mathbf{h}}_{n}+\widetilde{\mathbf{k}}_{n} e_{n},
$$

where $\widetilde{\mathbf{R}}_{n}=\sum_{j=1}^{n} \lambda^{n-j} \widetilde{\mathbf{x}}_{j} \widetilde{\mathbf{x}}_{j}^{T}$ and the modified Kalman gain is

$$
\widetilde{\mathbf{k}}_{n}=\widetilde{\mathbf{R}}_{n}^{-1} \widetilde{\mathbf{x}}_{n}=\frac{\lambda^{-1} \widetilde{\mathbf{R}}_{n-1}^{-1} \widetilde{\mathbf{x}}_{n}}{1+\lambda^{-1} \widetilde{\mathbf{x}}_{n}^{T} \widetilde{\mathbf{R}}_{n-1}^{-1} \widetilde{\mathbf{x}}_{n}},
$$

and using the matrix inversion lemma [1], $\widetilde{\mathbf{R}}_{n}^{-1}=\lambda^{-1}\left[\widetilde{\mathbf{R}}_{n-1}^{-1}-\right.$ $\left.\widetilde{\mathbf{k}}_{n} \widetilde{\mathbf{x}}_{n}^{T} \widetilde{\mathbf{R}}_{n-1}^{-1}\right]$. Although MMax-RLS updates all taps, complexity is reduced by the sparseness of $\widetilde{\mathbf{x}}_{n}$. The complexity in terms of multiplication and sorting operations for the MMax-RLS algorithm is $\mathcal{O}\left(L^{2}+M+2 \log _{2} L\right)$ compared to $\mathcal{O}\left(4 L^{2}\right)$ for RLS.

\section{ANALYSIS OF THE MISALIGNMENT}

We consider adaptive algorithms of the form

$$
\widehat{\mathbf{h}}_{n+1}=\widehat{\mathbf{h}}_{n}+\Gamma_{n} \mathbf{x}_{n} e_{n},
$$

where $\Gamma_{n}=2 \mu \mathbf{Q}_{n} /\left(\left\|\mathbf{x}_{n}\right\|_{2}^{2}+\delta\right)$ and $\Gamma_{n}=\widetilde{\mathbf{R}}_{n}^{-1} \mathbf{Q}_{n}$ are the $L \times L$ control matrices for MMax-NLMS and MMax-RLS respectively.

\subsection{Non-stationary system model}

A modified first-order Markov model [13][14] is employed to represent a time-varying unknown system

$$
\mathbf{h}_{n+1}=\xi \mathbf{h}_{n}+\sqrt{1-\xi^{2}} \mathbf{s}_{n}
$$

where $\mathbf{s}_{n}=\left[s_{n}(0), \ldots, s_{n}(L-1)\right]^{T}$ has a normal distribution $\mathcal{N}\left(0, \sigma_{s}^{2}\right)$ and $\sigma_{s}^{2}$ is the variance of $s_{n}$. As shown in [13], this model has the key features that (i) the parameter $0 \ll \xi<1$ controls the relative contributions to the instantaneous values of the coefficients of 'system memory' (the term $\xi \mathbf{h}_{n}$ ) and 'innovations' (the term $\sqrt{1-\xi^{2}} \mathbf{s}_{n}$ ), (ii) the average power of the coefficients is independent of $\xi$. It is shown in [13] that the system variation, which notionally indicates the difficulty of tracking by an adaptive filter, is a monotonic decreasing function of $\xi$. It has been found experimentally, that $\xi=0.9999$ is approximately equivalent to a source moving at $0.2 \mathrm{~ms}^{-1}$ for acoustic impulse responses $\mathbf{h}_{n}$ generated using the method of images [15].

Defining $E\{\cdot\}$ as the expectation operator, we first assume $E\left\{\mathbf{h}_{n}\right\}=0, E\left\{w_{n}\right\}=0$ and that $\mathbf{h}_{n}$ and $w_{n}$ are independent ${ }^{1}$. The dimension of $\widehat{\mathbf{h}}_{n}$ has been chosen to match the dimension of

${ }^{1}$ If $E\left\{\mathbf{h}_{n}\right\} \neq 0$, a bias will be induced in the variance of $\mathbf{h}_{n}$ by an amount proportional to the square value of $E\left\{\mathbf{h}_{n}\right\}$. $\mathbf{h}_{n}$. We then define the misalignment $\mathbf{v}_{n}=\widehat{\mathbf{h}}_{n}-\mathbf{h}_{n}$ which results in the error signal given by $e_{n}=w_{n}-\mathbf{x}_{n}^{T} \mathbf{v}_{n}$. Hence, we obtain

$$
\begin{aligned}
\mathbf{v}_{n+1}= & \widehat{\mathbf{h}}_{n+1}-\mathbf{h}_{n+1} \\
= & \mathbf{v}_{n}+(1-\xi) \mathbf{h}_{n}+\Gamma_{n} \mathbf{x}_{n} w_{n}-\sqrt{1-\xi^{2}} \mathbf{s}_{n}-\Gamma_{n} \mathbf{x}_{n} \mathbf{x}_{n}^{T} \mathbf{v}_{n} \\
\mathbf{R}_{\mathbf{v}, n+1}= & E\left\{\mathbf{v}_{n+1} \mathbf{v}_{n+1}^{T}\right\} \\
= & \mathbf{R}_{\mathbf{v}, n}+2(1-\xi) \sigma_{s}^{2} \mathbf{I}+\sigma_{w}^{2} E\left\{\Gamma_{n} \mathbf{x}_{n} \mathbf{x}_{n}^{T} \Gamma_{n}^{T}\right\} \\
& -\mathbf{R}_{\mathbf{v}, n} E\left\{\Gamma_{n} \mathbf{x}_{n} \mathbf{x}_{n}^{T}\right\}-\mathbf{R}_{\mathbf{v}, n} E\left\{\mathbf{x}_{n} \mathbf{x}_{n}^{T} \Gamma_{n}^{T}\right\} \\
& +E\left\{\Gamma_{n} \mathbf{x}_{n} \mathbf{x}_{n}^{T} \mathbf{v}_{n} \mathbf{v}_{n}^{T} \mathbf{x}_{n} \mathbf{x}_{n}^{T} \Gamma_{n}^{T}\right\}
\end{aligned}
$$

where $\mathbf{I}$ is the identity matrix and

$$
\begin{aligned}
E\left\{w_{n-i} w_{n-j}\right\} & = \begin{cases}\sigma_{w}^{2} & i=j \\
0 & \text { otherwise }\end{cases} \\
E\left\{\mathbf{v}_{n} \mathbf{v}_{n}^{T} \mathbf{x}_{n} \mathbf{x}_{n}^{T} \Gamma_{n}^{T}\right\} & =\mathbf{R}_{\mathbf{v}, n} E\left\{\mathbf{x}_{n} \mathbf{x}_{n} \Gamma_{n}^{T}\right\}
\end{aligned}
$$

and, from the definition of the first-order Markov model in (6), $E\left\{\mathbf{h}_{n} \mathbf{h}_{n}^{T}\right\}=E\left\{\mathbf{s}_{n} \mathbf{s}_{n}^{T}\right\}=\sigma_{s}^{2} \mathbf{I}$. Following the approach adopted in [1], we assume that the time variations of $\mathbf{h}_{n}$ are sufficiently slow that the adaptive filter is able to track the unknown system to within a time lag, and that after convergence, $\mathbf{v}_{n}$ is wide-sense stationary $\forall n$ so that $E\left\{\mathbf{v}_{n+1} \mathbf{v}_{n+1}^{T}\right\} \approx E\left\{\mathbf{v}_{n} \mathbf{v}_{n}^{T}\right\}=\mathbf{R}_{\mathbf{v}, n}$. We define $\mathbf{R}_{\mathbf{v}}$ as the autocorrelation matrix of the mean weight error vector which is approximately time-invariant under these assumptions. Defining the normalized misalignment as

$$
\eta=\left\|\mathbf{h}_{n}-\widehat{\mathbf{h}}_{n}\right\|_{2}^{2} /\left\|\mathbf{h}_{n}\right\|_{2}^{2}=\eta^{\prime} /\left\|\mathbf{h}_{n}\right\|_{2}^{2},
$$

the steady-state misalignment can then be expressed as $\eta^{\prime}=$ $\operatorname{tr}\left\{\mathbf{R}_{\mathbf{v}}\right\}$ where $\operatorname{tr}\{\cdot\}$ is the trace operator.

\subsection{Misalignment analysis of MMax-NLMS}

For misalignment, we consider (8) and evaluate $E\left\{\Gamma_{n} \mathbf{x}_{n} \mathbf{x}_{n}^{T}\right\}$. We note that elements $q_{n}(i), i=1, \ldots, L$, are not independent of $x_{n}(i)$ since only the $M$ largest $\left|x_{n}(i)\right|$ are selected. The $M$ selected samples are assumed to have zero mean and exploiting the mean ergodic theorem [1], the variance of $\widetilde{\mathbf{x}}_{n}$ is $\widetilde{\sigma}_{x}^{2}=L^{-1} \sum_{i=0}^{L-1} \widetilde{x}_{n}^{2}(i)$. Assuming that $\mathbf{x}_{n} \mathbf{x}_{n}^{T}$ is diagonal and using $E\left\{\mu_{n}\right\}=c$, a scalar constant, we can evaluate $E\left\{\Gamma_{n} \mathbf{x}_{n} \mathbf{x}_{n}^{T}\right\}=E\left\{\mathbf{x}_{n} \mathbf{x}_{n}^{T} \Gamma_{n}^{T}\right\}=$ $E\left\{\mu_{n}\right\} E\left\{\mathbf{Q}_{n} \mathbf{x}_{n} \mathbf{x}_{n}^{T}\right\}=\frac{M}{L} c \widetilde{\sigma}_{x}^{2} \mathbf{I}$. We can proceed to evaluate $\operatorname{tr}\left\{\mathbf{R}_{\mathbf{v}, n}\right\}$ using

$$
\begin{aligned}
& \operatorname{tr}\left\{E\left\{\Gamma_{n} \mathbf{x}_{n} \mathbf{x}_{n}^{T} \mathbf{v}_{n} \mathbf{v}_{n}^{T} \mathbf{x}_{n} \mathbf{x}_{n}^{T} \Gamma_{n}^{T}\right\}\right\} \\
& =\operatorname{tr}\left\{c^{2} E\left\{\mathbf{Q}_{n} \mathbf{x}_{n} \mathbf{x}_{n}^{T} \mathbf{v}_{n} \mathbf{v}_{n}^{T} \mathbf{x}_{n} \mathbf{x}_{n}^{T}\right\}\right\} \\
& =c^{2} \operatorname{tr}\left\{\mathbf{R}_{\mathbf{v}, n}\right\}(L+2) \frac{M}{L} \widetilde{\sigma}_{x}^{2} \sigma_{x}^{2}, \\
& \operatorname{tr}\left\{E\left\{\Gamma_{n} \mathbf{x}_{n} \mathbf{x}_{n}^{T} \Gamma_{n}^{T}\right\}\right\}=\operatorname{tr}\left\{\frac{M}{L} c^{2} \widetilde{\sigma}_{x}^{2} \mathbf{I}\right\}=\frac{M}{L} c^{2} \widetilde{\sigma}_{x}^{2} L .
\end{aligned}
$$

Substituting (10) into (8), we obtain

$$
\begin{aligned}
\operatorname{tr}\left\{\mathbf{R}_{\mathbf{v}, n+1}\right\}=\operatorname{tr} & \left\{\mathbf{R}_{\mathbf{v}, n}\right\}\left[1-2 \frac{M}{L} c \widetilde{\sigma}_{x}^{2}+(L+2) \frac{M}{L} c^{2} \widetilde{\sigma}_{x}^{2} \sigma_{x}^{2}\right] \\
& +M c^{2} \widetilde{\sigma}_{x}^{2} \sigma_{w}^{2}+2(1-\xi) L \sigma_{s}^{2} .
\end{aligned}
$$

The misalignment for MMax-NLMS can be found using the relation $E\left\{\mathbf{v}_{n+1} \mathbf{v}_{n+1}^{T}\right\} \approx E\left\{\mathbf{v}_{n} \mathbf{v}_{n}\right\}=\mathbf{R}_{\mathbf{v}, n}$ and $c=2 \mu /\left(L \sigma_{x}^{2}\right)$ hence giving the steady-state misalignment as

$$
\eta_{\mathrm{MMax}-\mathrm{NLMS}}^{\prime}=\frac{\mu \sigma_{w}^{2}}{\sigma_{x}^{2} \phi}+\frac{L \sigma_{x}^{2}}{\widetilde{\sigma}_{x}^{2} M} \frac{(1-\xi) L^{2} \sigma_{s}^{2}}{2 \mu \phi},
$$

where the term $\phi=1-c \sigma_{x}^{2}(1+0.5 L)$. Adopting the terminology of [9], the first term corresponds to the estimation variance 


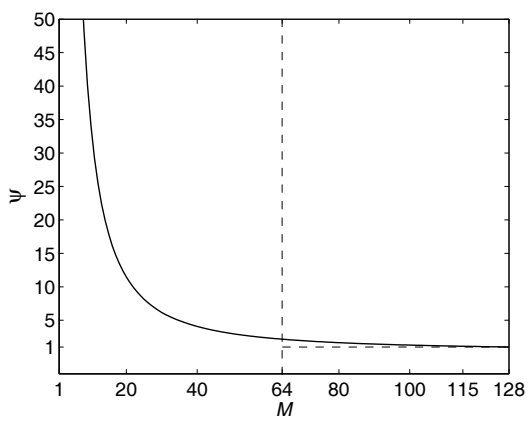

Fig. 2. Variation of $\psi$ with $M$ for $L=128$.

and is dependent on measurement noise $w_{n}$ while the second term corresponds to the lag variance and is due to system time variation $\xi$. We note that an additional factor of $L \sigma_{x}^{2} /\left(\widetilde{\sigma}_{x}^{2} M\right)$ arises in the lag variance for MMax-NLMS compared ${ }^{2}$ to NLMS. To quantify the closeness of tap selection to that of a full tap-input vector in an MMax sense, we employ the M-ratio [7] given by $\mathcal{M}=$ $\left\|\mathbf{Q}_{n} \mathbf{x}_{n}\right\|_{2}^{2} /\left\|\mathbf{x}_{n}\right\|_{2}^{2}=\widetilde{\sigma}_{x}^{2} / \sigma_{x}^{2}$. As shown in [7], $\mathcal{M}$ exhibits only a modest reduction for $0.5 L \leq M<L$ and hence a graceful reduction in convergence rate is expected over this range of $M$ as compared to fully updated NLMS. The steady-state misalignment due to tap selection $M$ is apparent by noting from (12) that, under a time-varying unknown system condition $\xi<1$, the lag variance is proportional to

$$
\psi=L \sigma_{x}^{2} /\left(\tilde{\sigma}_{x}^{2} M\right)=L /(M \mathcal{M}) .
$$

Figure 2, shows the variation of $\psi$ with the number of selected taps $M$ for an example case of $L=128$ using a zero mean, unit variance white Gaussian noise (WGN) input sequence. We note that for $M=L, \psi=1$ since $\widetilde{\sigma}_{x}^{2}=\sigma_{x}^{2}$ and $\mathcal{M}=1$. More importantly, $\psi$ increases smoothly with reducing $M$ within the region $0.5 L \leq M<L$ and hence, for this range of $M$, we would expect only a graceful degradation in steady-state misalignment performance for time-varying case $\xi<1$. This is consistent with results shown in [4] for a stationary system when $\xi=1$.

\subsection{Misalignment analysis of MMax-RLS}

Using (3) and (4), the tap update equation for the MMax-RLS algorithm can be expressed as

$$
\widehat{\mathbf{h}}_{n+1}=\widehat{\mathbf{h}}_{n}+\widetilde{\mathbf{R}}_{n}^{-1} \mathbf{Q}_{n} \mathbf{x}_{n} e_{n} .
$$

where $\Gamma_{n}=\widetilde{\mathbf{R}}_{n}^{-1} \mathbf{Q}_{n}$ in (5) and $\widetilde{\mathbf{R}}_{n}=\sum_{j=1}^{n} \lambda^{n-j} \mathbf{Q}_{j} \mathbf{x}_{j} \mathbf{x}_{j}^{T} \mathbf{Q}_{j}^{T}$. For $n \rightarrow \infty$,

$$
\begin{aligned}
E\left\{\lim _{n \rightarrow \infty} \widetilde{\mathbf{R}}_{n}\right\} & =E\left\{\lim _{n \rightarrow \infty}\left(\lambda^{n-1} \widetilde{\mathbf{x}}_{1} \widetilde{\mathbf{x}}_{1}^{T}+\ldots+\widetilde{\mathbf{x}}_{n} \widetilde{\mathbf{x}}_{n}^{T}\right)\right\} \\
& =\frac{1}{1-\lambda} \frac{M \widetilde{\sigma}_{x}^{2}}{L} \mathbf{I},
\end{aligned}
$$

and hence $\Gamma_{n}=(1-\lambda)\left(L /\left(M \widetilde{\sigma}_{x}^{2}\right)\right) \mathbf{Q}_{n}$. We may express the following terms in (8) as

$$
\begin{aligned}
E\left\{\Gamma_{n} \mathbf{x}_{n} \mathbf{x}_{n}^{T}\right\}=E\left\{\mathbf{x}_{n} \mathbf{x}_{n}^{T} \Gamma_{n}^{T}\right\} & =(1-\lambda) \frac{L}{M \widetilde{\sigma}_{x}^{2}} E\left\{\mathbf{Q}_{n} \mathbf{x}_{n} \mathbf{x}_{n}^{T}\right\} \\
& =(1-\lambda) \mathbf{I}
\end{aligned}
$$

\footnotetext{
${ }^{2}$ The steady-state misalignment for NLMS can be found by letting $M=$ $L$ and $\widetilde{\sigma}_{x}^{2}=\sigma_{x}^{2}$.
}

$$
\begin{aligned}
E\left\{\Gamma_{n} \mathbf{x}_{n} \mathbf{x}_{n}^{T} \mathbf{v}_{n} \mathbf{v}_{n}^{T} \mathbf{x}_{n} \mathbf{x}_{n}^{T} \Gamma_{n}^{T}\right\}= & {\left[\frac{(1-\lambda) L}{M \widetilde{\sigma}_{x}^{2}}\right]^{2} \times } \\
& E\left\{\mathbf{Q}_{n} \mathbf{x}_{n} \mathbf{x}_{n}^{T} \mathbf{x}_{n} \mathbf{x}_{n}^{T} \mathbf{Q}_{n}\right\} \mathbf{R}_{\mathbf{v}, n} \\
= & \frac{(1-\lambda)^{2} L(L+2) \sigma_{x}^{2}}{M \widetilde{\sigma}_{x}^{2}} \mathbf{R}_{\mathbf{v}, n}, \\
E\left\{\Gamma_{n} \mathbf{x}_{n} \mathbf{x}_{n}^{T} \Gamma_{n}^{T}\right\}= & {\left[\frac{(1-\lambda) L}{M \widetilde{\sigma}_{x}^{2}}\right]^{2} E\left\{\mathbf{Q}_{n} \mathbf{x}_{n} \mathbf{x}_{n}^{T} \mathbf{Q}_{n}\right\} } \\
= & \frac{(1-\lambda)^{2} L}{M \widetilde{\sigma}_{x}^{2}} \mathbf{I} .
\end{aligned}
$$

Using the same approach as (11), by substituting the set of equations in (17) into (8),

$$
\begin{aligned}
\mathbf{R}_{\mathbf{v}, n+1}= & \mathbf{R}_{\mathbf{v}, n}-2(1-\lambda) \mathbf{R}_{\mathbf{v}, n}+\frac{(1-\lambda)^{2} L}{M \widetilde{\sigma}_{x}^{2}} \sigma_{w}^{2} \mathbf{I} \\
& +\frac{(1-\lambda)^{2} L(L+2) \sigma_{x}^{2}}{M \widetilde{\sigma}_{x}^{2}} \mathbf{R}_{\mathbf{v}, n}+2(1-\xi) \sigma_{s}^{2} \mathbf{I} .
\end{aligned}
$$

As before, for $n \rightarrow \infty, \mathbf{R}_{\mathbf{v}, n+1} \approx \mathbf{R}_{\mathbf{v}, n}=\mathbf{R}_{\mathbf{v}}$. Defining $\eta^{\prime}=$ $\operatorname{tr}\left\{\mathbf{R}_{\mathbf{v}}\right\}$ we may then express (18) as

$$
\eta_{\mathrm{MMax}-\mathrm{RLS}}^{\prime}=\frac{(1-\lambda) L^{2} \sigma_{w}^{2}}{\beta}+\frac{2 L(1-\xi) \sigma_{s}^{2} M \widetilde{\sigma}_{x}^{2}}{(1-\lambda) \beta},
$$

where $\beta=L \sigma_{x}^{2}\left[2 \psi^{-1}-(1-\lambda)(L+2)\right]$ while $\psi$ is defined in (13). For $M=L$ and $\widetilde{\sigma}_{x}^{2}=\sigma_{x}^{2}, \eta_{\text {MMax-RLS }}^{\prime}$ is equivalent to the steadystate misalignment performance of RLS. More importantly, the estimation variance for MMax-RLS is dependent on $M$. As can be seen, $\beta$ is a decreasing function of $\psi$ and hence for a time-invariant system with $\xi=1$, the steady-state misalignment $\eta_{\mathrm{MMax}-\mathrm{RLS}}^{\prime}$ is a decreasing function of $M$. This is contrary to the MMax-NLMS case as shown in (12) where the steady-state normalized misalignment is independent of $M$ for time-invariant systems.

\section{SIMULATION RESULTS}

We present simulation results to support the analysis of our derivations and compare the performances of MMax-NLMS and MMaxRLS to that of NLMS and RLS under time-varying system conditions. Employing misalignment $\eta$ as defined in (9), Figure 3 shows MMax-NLMS results for a time-invariant $(\xi=1)$ and three timevarying systems $(\xi=0.999999,0.99999,0.9999)$ where smaller values of $\xi$ indicate higher degrees of time-variation. An adaptive filter of length $L=1024$ is used with $M=128$ and a step-size of $\mu=$ 0.7. An additive white Gaussian noise (WGN) $w_{n}$ is added to $y_{n}$ to achieve an SNR of $30 \mathrm{~dB}$. The learning curves are averaged over 3 trials and the theoretical misalignment values of $\eta_{\mathrm{MMax}-\mathrm{NLMS}}$ given by (9) and (12) are plotted as horizontal solid lines. Theoretical misalignment values of $\eta_{\text {NLMS }}$ are plotted as dashed lines for comparison. The results show that for both NLMS and MMax-NLMS, their misalignment performance degrades with reducing $\xi$. The MMaxNLMS algorithm perform around 3 to $4 \mathrm{~dB}$ worse, in terms of steady-state normalized misalignment, than NLMS for $\xi<1$. For a time-invariant system, $\xi=1, \eta_{M M a x-N L M S}=\eta_{N L M S}$ since from (12), their estimation variances are equivalent.

Figure 4 shows MMax-RLS results for the same unknown system conditions. The adaptive filter is of length $L=1024$ with $M=128$ while the forgetting factor is $\lambda=1-1 /(3 L)$ and the SNR is $30 \mathrm{~dB}$. The theoretical misalignment values of $\eta_{\text {MMax }}-\mathrm{RLS}$ given by (9) and (19) are plotted as straight horizontal solid lines. In addition, the theoretical misalignment values of $\eta_{\mathrm{RLS}}$ computed using $M=L$ are plotted as dashed lines for comparison. As before, we note that for both RLS and MMax-RLS, their misalignment performance degrades with increasing deviation of $\xi$ from unity. Unlike 


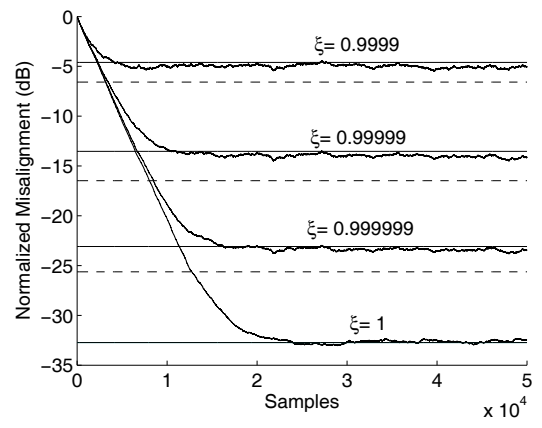

Fig. 3. Theoretical normalized misalignment for NLMS (dashed) and MMax-NLMS (solid) $[L=1024, M=128, \mu=0.7, \mathrm{SNR}=30 \mathrm{~dB}]$.

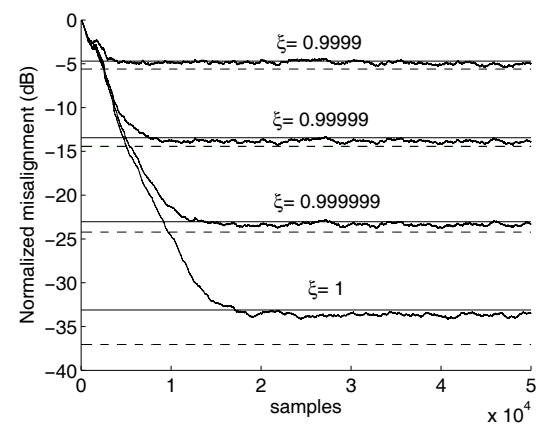

Fig. 4. Theoretical normalized misalignment for RLS (dashed) and MMaxRLS (solid) $[L=1024, M=128, \lambda=1-1 /(3 L), \mathrm{SNR}=30 \mathrm{~dB}]$.

the case of MMax-NLMS, there is a degradation in misalignment performance of $\sim 5 \mathrm{~dB}$ for MMax-RLS compared to RLS even for a time-invariant system $\xi=1$.

Fig. 5 shows the tradeoff between complexity reduction and degradation in misalignment performance for MMax-NLMS in a time-varying system condition of $\xi=0.999999$ over a range of $L / 8 \leq M \leq L$ for $L=1024$. The theoretical and experimental steady-state misalignment is plotted against the number of multiplications assuming the sorting complexity is $2 \log _{2} L=20$ as in the SORTLINE algorithm [11]. The experimental curves are consistent with the theoretical results to within about $0.5 \mathrm{~dB}$ for this time-varying case of unknown system.

\section{CONCLUSION}

We have presented an analytical framework to compare the performance of MMax-NLMS and MMax-RLS with NLMS and RLS respectively under time-varying unknown system conditions. Insights into the performance of MMax-NLMS and MMax-RLS is provided through this mathematical analysis. It is shown that under timeinvariant system conditions, the MMax-NLMS has the same steadystate normalized misalignment as NLMS. Under time-varying system conditions, degradation in misalignment performance is found and quantified. The performance in steady-state misalignment for MMax-RLS is degraded compared to RLS even under time-invariant system conditions. The tradeoff between computational complexity and normalized misalignment indicates the MMax tap-selection does not degrade convergence significantly for $M=L / 2$ even for a timevarying unknown system whereas $M=L / 8$ costs an additional 2 to $2.5 \mathrm{~dB}$ in misalignment whilst saving a further $43 \%$ of the multi-

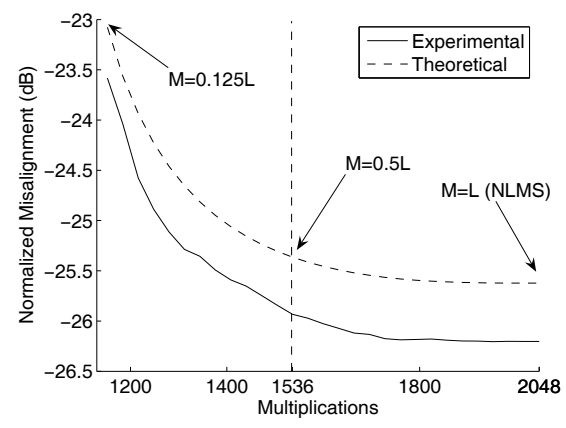

Fig. 5. Tradeoff between complexity and normalized misalignment for $L=$ $1024, \xi=0.999999, \mathrm{SNR}=30 \mathrm{~dB}$.

plications. Our new analysis results are verified by simulations.

\section{REFERENCES}

[1] S. Haykin, Adaptive Filter Theory, 4th ed., ser. Information and System Science. Prentice Hall, 2002.

[2] S. C. Douglas, "Adaptive filters employing partial updates," IEEE Trans. Circuits Syst. II, vol. 44, no. 3, pp. 209-216, Mar. 1997.

[3] T. Aboulnasr and K. Mayyas, "MSE analysis of the M-Max NLMS adaptive algorithm," in Proc. IEEE Int. Conf. Acoustics Speech Signal Processing, vol. 3, 1998, pp. 1669-1672.

[4] P. A. Naylor and A. W. H. Khong, "Affine projection and recursive least squares adaptive filters employing partial updates," in Proc. Thirty-Eighth Asilomar Conference on Signals, Systems and Computers, vol. 1, Nov. 2004, pp. 950-954.

[5] K. Dogancay and O. Tanrikulu, "Adaptive filtering algorithms with selective partial updates," IEEE Trans. Circuits Syst. II, vol. 48, no. 8, pp. 762-769, Aug. 2001.

[6] S. L. Gay and S. Tavathia, "The fast affine projection algorithm," in Proc. IEEE Int. Conf. Acoustics Speech Signal Processing, vol. 5, 1995, pp. 3023-3026.

[7] A. W. H. Khong and P. A. Naylor, "Selective-tap adaptive algorithms in the solution of the non-uniqueness problem for stereophonic acoustic echo cancellation," IEEE Signal Processing Lett., vol. 12, no. 4, pp. 269-272, Apr. 2005

[8] B. Widrow and E. Walach, "On the statistical efficiency of the LMS algorithm with nonstaionary inputs," IEEE Trans. Inform. Theory, vol. 30, no. 2, pp. 211-221, 1984.

[9] O. Macchi, "Optimization of adaptive identification for time-varying filters," IEEE Trans. Automat. Contr., vol. 31, no. 3, pp. 283-287, 1986.

[10] T. Aboulnasr and K. Mayyas, "Selective coefficient update of gradientbased adaptive algorithms," in Proc. IEEE Int. Conf. Acoustics Speech Signal Processing, vol. 3, 1997, pp. 1929-1932.

[11] I. Pitas, "Fast algorithms for running ordering and $\mathrm{max} / \mathrm{min}$ calculation," IEEE Trans. Circuits Syst., vol. 36, no. 6, pp. 795-804, Jun. 1989

[12] P. A. Naylor and W. Sherliker, "A short-sort M-max NLMS partial update adaptive filter with applications to echo cancellation," in Proc. IEEE Int. Conf. Acoustics Speech Signal Processing, vol. 5, 2003, pp. 373-376.

[13] N. J. Bershad, S. McLaughlin, and C. F. N. Cowan, "Performance comparison of RLS and LMS algorithms for tracking a first order Markov communications channel," in Proc. IEEE Int. Symposium on Circuits and Systems, vol. 1, 1990, pp. 266-270.

[14] W. Sherliker, "Acoustic echo cancellation algorithms with tap selection for non-stationary environments," Ph.D. dissertation, Imperial College London, 2000.

[15] J. B. Allen and D. A. Berkley, "Image method for efficiently simulating small-room acoustics," J. Acoust. Soc. Amer, vol. 65, no. 4, pp. 943950, Apr. 1979 